\title{
O Perdão Interpessoal em Relação a Variáveis Psicossociais e Demográficas
}

\author{
Interpersonal Forgiveness in Relation to Psychosocial \\ and Demographic Variables
}

\author{
Júlio Rique* \& Cleonice Pereira Santos Camino \\ Universidade Federal da Paraíba, João Pessoa, Brasil
}

\begin{abstract}
Resumo
O perdão foi estudado através do Enright Forgiveness Inventory - EFI (versão em português) - em relação à intensidade da mágoa, aos afetos, aos julgamentos e aos comportamentos da vítima referentes ao ofensor. Verificou-se também se a cultura, o sexo, a idade e a religião influenciam o perdão. Participaram 200 brasileiros e 394 estadunidenses. Os resultados foram similares nas duas culturas: as pessoas perdoaram espontaneamente, mas não completamente. O perdão mostrou-se negativamente correlacionado com a intensidade da mágoa; foi oferecido em maior grau à família e aos amigos do que a estranhos e a colegas de trabalho; e as pessoas perdoaram mais pelos julgamentos do que pelos afetos. Não ocorreram diferenças quanto à religião, sexo ou idade. Futuros estudos devem verificar se ao perdoar completamente os afetos positivos pelo ofensor permanecem inferiores ou se igualam aos escores dos julgamentos positivos. Palavras-chave: Perdão interpessoal; Intensidade da mágoa; Atitude moral.
\end{abstract}

\begin{abstract}
The Enright Forgiveness Inventory - EFI (Portuguese version) was used to verify relationships between forgiveness and degree of injury to the agents of hurt and also the degree of affect, behavior and judgment from victims towards their offenders. It was also checked whether culture, gender, age and religion affect the act of forgiving. Two hundred Brazilians and 394 Americans participated in the study. Findings were similar for both cultures. Forgiveness was negatively related to the degree of injury. People forgive family and friends at a higher degree than they forgive strangers or co-workers. Positive judgments received significantly higher scores than positive affect. No significant effect was found for forgiveness regarding gender, age or religion. Future studies should verify whether genuine forgiveness shows positive affect for offenders at a same degree as positive judgments or affect remains always lower than judgment for forgiving.

Keywords: Interpersonal forgiveness; Degree of injury; Attitude moral.
\end{abstract}

Ao longo da história da civilização, o perdão foi enfocado como uma estratégia política para a resolução de conflitos (Arendt, 1958/1998; Digeser, 2001; McKnight, 2004; Nussbaum, 1998). Worthington $(1998,2005)$ infor-

\footnotetext{
${ }^{*}$ Endereço para correspondência: Universidade Federal da Paraíba, Departamento de Psicologia, Cidade Universitária, Campus I, Castelo Branco, João Pessoa, PB, Brasil, CEP 58051-900. Tel.: (083) 3216 7337. E-mail: julio.rique@uol.com.br

Esta pesquisa foi realizada como parte da tese de doutorado do primeiro autor, na área de Desenvolvimento Humano, na Universidade de Wisconsin, Madison, nos Estados Unidos. Os autores agradecem aos professores Robert D. Enright, $\mathrm{PhD}$ e Michael Subkoviak, $\mathrm{PhD}$, por disponibilizarem o banco de dados americanos e pela orientação da tese de doutorado do primeiro autor. Os autores agradecem também a contribuição dos revisores anônimos, cujos comentários contribuíram para o trabalho.

Em particular, o primeiro autor agradece o apoio recebido da Coordenação de Aperfeiçoamento de Pessoal de Nível Superior (CAPES), através da concessão de uma Bolsa para Estudos de Doutorado.
}

ma que, no âmbito da psicologia, os estudos sobre o perdão são recentes. As publicações sobre o tema começaram a despontar em 1968 e mostraram um crescimento significativo durante as décadas de 1980 e 1990, permanecendo o interesse relativamente alto desde então.

No terreno da psicologia, um campo de estudos sobre o perdão tem sido o das relações interpessoais. Nesse campo, o perdão é operacionalizado como uma atitude motivada pela compaixão e observado em suas implicações para o desenvolvimento moral e social. Neste sentido, encontram-se os estudos de Robert D. Enright, com um corpo teórico e metodológico coerentemente aplicado a pesquisas empíricas em diversas áreas: psicologia do desenvolvimento moral (Enright \& The Human Development Study Group, 1991, 1994), avaliação psicológica (Rique, Camino, Santos, \& Gouveia, 2009; Subkoviak et al., 1995), psicologia clinica (Enright \& Fitzgibbons, 2001; Reed \& Enright, 2006) e psicologia social (Holter, Martin, \& Enright, 2003). 
Na psicologia do desenvolvimento, os estudos sobre raiva, depressão e ressentimento recebem muito mais ênfase do que os estudos sobre as experiências positivas no desenvolvimento ou as emoções positivas como a compaixão (Seligman \& Csikszentmihalyi, 2000). Essa preferência pelas emoções retributivas negativas é decorrente das tradições psicanalítica e naturalista, nas quais os traumas, desejos recalcados, ressentimentos e raivas são vistos como componentes estruturais e motivacionais para o desenvolvimento. Diferentemente da raiva ou do ressentimento, o perdão como uma expressão da compaixão não é entendido como uma resposta natural, mas como uma construção moral do cristianismo, um ato de fé ou de religiosidade. O perdão também é visto como um valor que, se for internalizado, vai interferir nos sentimentos morais naturais de raiva ou ressentimento (Murphy \& Hampton, 1988/1998). Mesmo na literatura cristã ortodoxa, Joseph Butler, Arcebispo de Canterbury, em seus Sermões (Butler, 1827/2002), ao especular sobre a natureza da condição humana, concluiu que, para motivar respostas às injustiças, Deus proveu o homem com o ressentimento e não com a compaixão. Segundo Butler (1827/2002), ao invés de restaurar no organismo magoado a capacidade natural de indignação e de ressentimento, o perdão elimina esta possibilidade. Atualmente, na abordagem do desenvolvimento humano, muitos autores discordam de tanta temeridade relacionada ao perdão e julgam necessário estudar empiricamente como ele ocorre (Worthington, 2005).

Em seus estudos, Enright procura enfocar o perdão interpessoal a partir da perspectiva das vítimas (Enright \& Fitzgibbons, 2001; Enright, Freedman, \& Rique, 1998). Segundo esse pesquisador, o perdão interpessoal pode ser definido como:

... uma atitude moral na qual uma pessoa considera abdicar do direito ao ressentimento, julgamentos negativos, e comportamentos negativos para com a pessoa que ofendeu injustamente. E, ao mesmo tempo, nutrir a compaixão, a misericórdia, e possivelmente o amor para com o ofensor. (Enright et al., 1998, p. 46-47, tradução nossa).

Quanto ao processo psicológico do perdão, tomandose a perspectiva da vítima com relação ao ofensor, o perdão corresponde a uma diminuição dos julgamentos negativos (ex., ela é uma pessoa má), dos sentimentos negativos (ex., raiva) e dos comportamentos negativos (ex., indiferença, agressões verbais) com relação ao outro que ofendeu. Ao mesmo tempo, corresponde a um aumento dos sentimentos positivos (ex., carinho, boa vontade), dos julgamentos positivos (ex., ela é uma boa pessoa) e dos comportamentos positivos (ex., ajudar quando ela estiver com problemas). Esses processos são complementares e indissociáveis. A redução dos elementos negativos sem o aumento dos elementos positivos não significa o perdão genuíno, mas, provavelmente, uma etapa do processo (Enright \& The Human Development Study Group, 1991).
Para perdoar é preciso, primeiramente, que uma pessoa reconheça que foi magoada por outra pessoa. A mágoa é uma função da percepção do evento que gerou o sentimento de injustiça. Assim, essa mágoa vai variar de intensidade mesmo entre pessoas que vivenciaram injustiças semelhantes. Quanto maior a percepção de uma mágoa, mais a pessoa é levada a focalizar sua atenção na raiva, no ressentimento e na indignação. Portanto, é esperado que a percepção da mágoa seja negativamente correlacionada com o grau do perdão.

As pesquisas indicam que os contextos mais reportados com relação às mágoas se referem a conflitos normativos no desenvolvimento humano. Ou seja, dizem respeito às mágoas consideradas comuns nos relacionamentos sociais na família, nas amizades e no trabalho. Frequentemente, as mágoas (ex., agressões verbais e traições) são reportadas como ocorrendo entre familiares, esposos, amigos e namorados, ou seja, pessoas muito próximas das vitimas (Rique, Camino, Enright, \& Queiroz, 2007).

Como todo trabalho empírico requer medidas de avaliação válidas e confiáveis, Subkoviak et al. (1995) criaram a escala denominada Enright Forgiveness Inventory (EFI). Elaborada segundo a definição do perdão interpessoal, a EFI avalia o grau com o qual a vítima perdoa o ofensor (em uma escala Lickert de 5 pontos) em três subescalas: afeto, comportamento e julgamento. A soma dos escores das três subescalas indica o grau ou a atitude para com o perdão. A EFI é bastante utilizada em pesquisas sobre o grau do perdão em diferentes contextos de mágoas e foi traduzida e validada para o português com o título de Escala de Atitudes para o Perdão ([EFI], Enright \& Rique, 2001; Rique, 1999).

Rique et al. (in press) fizeram um estudo detalhado das qualidades psicométricas da EFI, utilizando uma amostra de 271 adolescentes, de ambos os sexos, com idade média de 15 anos, residentes na cidade de João Pessoa-Pb. Nessa amostra, foram testados três modelos possíveis do processo psicológico avaliado pela EFI: (a) o modelo unifatorial, no qual o perdão possui um único fator, que não discrimina significativamente os afetos, comportamentos e julgamentos; (b) o modelo tripartite, no qual o perdão é composto por três fatores de primeira ordem: afeto, comportamento e julgamento; e, por último, (c) o modelo de perdão com seis fatores de primeira ordem: afetos positivos e negativos, comportamentos positivos e negativos e julgamentos positivos e negativos.

Os resultados mostraram que o modelo que considerou o perdão constituído por seis fatores de primeira ordem foi o que melhor se ajustou aos dados. Considerando ainda que os seis fatores sejam independentes, embora bastante correlacionados, o procedimento parcimonioso de agrupar os resultados das subescalas dentro das três dimensões de afeto, comportamento e julgamento mostrou-se adequado e foi defendido pelos autores. Em outras palavras, o grau do perdão representado pelo escore total da EFI indica a presença de afetos positivos, 
comportamentos positivos e julgamentos positivos, e a ausência de afetos negativos, comportamentos negativos e julgamentos negativos para um ofensor.

Além do português, a EFI foi também traduzida e validada para mais quatro idiomas, o alemão, o holandês, o hebreu e o norueguês, com normas disponíveis para diversas culturas. Pesquisas nessas culturas (Enright \& Fitzgibbons, 2001) mostram que a EFI é válida e possui forte consistência interna: Áustria (versão em alemão, $\propto=0,98$ ), Brasil (versão em português, $\propto=0,98$ ), Bélgica (versão em holandês, $\propto=0,98$ ), Coréia (versão em inglês, $\propto=0,97$ ), Estados Unidos (original em inglês, $\propto=0,98$ ), Israel (versão em hebreu, $\propto=0,98$ ), Noruega (versão em norueguês, $\propto=0,98$ ) e Taiwan (versão em inglês, $\propto=0,97$ ).

Subkoviak et al. (1995) utilizaram a escala EFI para estudar o grau do perdão em relação ao grau de mágoa em adultos e jovens estudantes universitários americanos. A amostra era relacionada, no sentido de que os adultos eram pais dos jovens estudantes, os quais eram do mesmo sexo dos pais que foram entrevistados. Os resultados mostraram uma correlação negativa significante, indicando que, quanto maior a intensidade da mágoa, menor era o perdão. Os resultados também mostraram um efeito significativo da idade no grau do perdão: um maior grau de perdão foi encontrado na amostra dos pais, em comparação com a amostra dos filhos. Não foram encontradas diferenças significativas entre os sexos, em ambas as amostras.

Por outro lado, Park (1998) empreendeu um estudo com uma amostra de 112 adultos e jovens universitários americanos utilizando uma metodologia semelhante à de Subkoviak et al. (1995). Nos seus resultados, encontrou uma interação significativa entre idade e sexo, indicando que: as mães $(n=28)$ perdoaram mais do que os pais ( $n=28)$ e os homens jovens universitários perdoaram ( $n=28)$ mais do que as mulheres jovens universitárias $(n=28)$.

Portanto, as pesquisas de Park (1998) e Subkoviak et al. (1995), que utilizaram um delineamento metodológico semelhante, indicam uma variação no grau do perdão com relação ao sexo dos participantes e à idade. Dessa maneira, torna-se inconclusiva, na literatura especializada sobre o tema, uma posição sobre o efeito dessas variáveis no perdão. Por último, Park (1998) e Subkoviak et al. (1995) reportam que os estudos não encontraram correlações entre o perdão e a desejabilidade social medida pela escala de Crowne e Marlowe (1960).

Em resumo, a revisão da literatura sobre as pesquisas a respeito do perdão interpessoal, na abordagem de Enright, indica que o grau do perdão é influenciado pela intensidade da mágoa sofrida e pela proximidade entre as vítimas e os ofensores, em amostras de jovens universitários e adultos americanos de classe média. Quanto ao grau de perdão com relação à idade, ao sexo, ao tempo decorrido desde a mágoa e à religião, os estudos não permitem extrair conclusões gerais, talvez porque o perdão seja influenciado por contextos específicos e por percepções diferentes das mágoas.

Apesar da escala do perdão EFI já estar validada para o português e da importância de estudos empíricos sobre o tema, não existem pesquisas no Brasil sobre o perdão utilizando essa escala. É em função dessa ausência de investigações que se justifica a realização do presente trabalho, com o objetivo principal de verificar o perdão interpessoal, utilizando a EFI com participantes brasileiros. Além disso, torna-se relevante uma comparação dos resultados obtidos no Brasil com aqueles produzidos nos Estados Unidos, a fim de contribuir para o avanço das pesquisas e para a resolução das questões ainda pendentes na área. Ressalte-se que a ênfase nesse estudo é entender o perdão na perspectiva do desenvolvimento humano em cada cultura, ao invés de se comparar o perdão entre culturas.

De maneira mais específica, os objetivos desta pesquisa podem são: (a) verificar se as pessoas perdoam suas mágoas interpessoais espontaneamente e de forma completa; (b) verificar se o grau do perdão varia em relação à intensidade da mágoa sofrida e da proximidade no relacionamento com o ofensor. Ou seja, determinar até que ponto se perdoa mais os familiares e os amigos do que os estranhos e os colegas de trabalho; (c) investigar se a cultura, o sexo, a idade e a afiliação religiosa das vítimas influenciam o grau do perdão; e (d) delimitar como o perdão se expressa naqueles que sofreram uma grande mágoa, em relação aos afetos, julgamentos e comportamentos para com a pessoa que os ofendeu.

\section{Método}

Este estudo teve um delineamento correlacional ex-postfacto. O grau de perdão foi a variável de critério, enquanto que a cultura, a afiliação religiosa, a intensidade da mágoa e a proximidade com o/a ofensor/a constituíram as variáveis de predição.

\section{Participantes}

Participaram deste estudo 200 brasileiros e 394 americanos, conforme descrito na Tabela 1.

\section{Instrumentos}

Informações Demográficas. Foi utilizado um questionário para obter informações sobre idade, sexo, nível educacional e afiliação religiosa dos participantes.

A Escala de Atitudes para o Perdão ([EFI], Enright Forgiveness Inventory). A EFI possui três partes, com instruções apresentadas no início de cada parte. A primeira parte é formada por uma Folha de Rosto, que coleta informações sobre a vítima e sobre a ofensa. Também consta da primeira parte um item que solicita a cada participante: (a) lembrar de uma situação na qual, injustamente, foi ofendido por outra pessoa; (b) focalizar a 
Tabela 1

Informações Bio-Sócio-Demográfica dos Participantes

\begin{tabular}{|c|c|c|c|c|c|c|c|c|c|c|}
\hline \multirow[t]{2}{*}{ Variáveis } & \multicolumn{3}{|c|}{ Idade } & \multicolumn{2}{|c|}{ Sexo } & \multicolumn{2}{|c|}{ Educação } & \multicolumn{3}{|c|}{$\begin{array}{c}\text { Possui Afiliação } \\
\text { Religiosa? }\end{array}$} \\
\hline & $\begin{array}{l}\text { Jovens } \\
n(M-d p)\end{array}$ & $\begin{array}{l}\text { Adultos } \\
\qquad n(M-d p)\end{array}$ & $\begin{array}{l}\text { Total } \\
N(\max .-\min .)\end{array}$ & $\begin{array}{l}\mathrm{H} \\
\%\end{array}$ & $\begin{array}{l}\mathrm{M} \\
\%\end{array}$ & $\begin{array}{c}\text { Fund. } \\
\%\end{array}$ & $\begin{array}{c}\text { Médio } \\
\%\end{array}$ & $\begin{array}{c}\text { Sup. } \\
\%\end{array}$ & $\begin{array}{c}\mathrm{Sim} \\
\%\end{array}$ & $\begin{array}{c}\text { Não } \\
\%\end{array}$ \\
\hline Brasil & $100(23-3,8)$ & $100(50-7,7)$ & $200(18-72)$ & 50 & 50 & 19 & 60,5 & 20,5 & 84,5 & 15,5 \\
\hline Estados Unidos & $197(22-4,7)$ & $197(50-9,6)$ & $394(19-99)$ & 48 & 52 & 02 & 63 & 35 & 70 & 30 \\
\hline
\end{tabular}

atenção na pessoa que ofendeu (considerando o momento presente) e relatar: o grau da mágoa em uma escala de 5 pontos (1- nenhuma mágoa, 5- uma grande mágoa) e o agente da mágoa (pais, filhos, familiares, amigos, colegas de trabalho ou outros).

Na segunda parte, a EFI apresenta um inventário composto por adjetivos, substantivos e pequenas sentenças, medidos através de uma escala de concordância objetiva, com 60 itens colocados em uma escala de 6 pontos (1- discordo fortemente, 6- concordo fortemente). Os itens se dividem em afetos negativos (10 itens, ex., amargura), afetos positivos (10 itens, ex., carinho), comportamentos negativos (10 itens, ex., falar mal dele ou dela), comportamentos positivos (10 itens, ex., mostrar amizade), julgamentos negativos (10 itens, ex., corrupto) e julgamentos positivos (10 itens, ex., de boas qualidades). Os escores dos itens negativos são revertidos e agrupados em três grandes grupos: julgamentos, comportamentos e afetos. Os escores internos dessas subescalas da EFI podem ser interpretados de duas maneiras: (a) pelo grau de presença dos afetos, comportamentos e julgamentos positivos em relação à pessoa que ofendeu; e (b) pelo grau de ausência dos afetos, comportamentos e julgamentos negativos em relação à pessoa que ofendeu. $\mathrm{O}$ manual de uso da EFI (Enright \& Rique, 2001) informa que não se deve usar apenas escores de uma subescala em particular. Por exemplo, não se deve usar apenas escores de afetos positivos, pois, teoricamente, o perdão se processa como uma atitude homogênea, na qual a valência dos afetos é associada à valência dos julgamentos e dos comportamentos. O escore total da EFI é fornecido pela soma dos escores de cada subescala, ou seja, a soma dos 60 itens indicando o grau de atitude para o perdão. $\mathrm{O}$ escore total varia de 60 (baixo grau de perdão) a 360 (alto grau de perdão).

A terceira parte é constituída por uma medida independente, um item que pede a cada participante que informe o quanto ele ou ela perdoou a pessoa avaliada na EFI. Esse item possui uma escala de 5 pontos (1- não perdoei, 3- estou considerando o perdão, 5- perdoei completamente), que pode ser administrada em separado ou como uma última questão da EFI. É esperado que o item do perdão se correlacione positivamente com o escore total da EFI.
A escala EFI apresenta forte índice de confiabilidade interna $(\propto=0,98)$ nas amostras brasileiras e americanas. As propriedades psicométricas da EFI encontramse descritas com maiores detalhes em Rique et al. (in press).

Desejabilidade Social (Crowne \& Marlowe, 1960, versão de Rique, 1999). Esta é uma escala objetiva, com 33 itens, na qual os participantes devem marcar como Verdadeiro ou Falso cada item sobre comportamentos sociais. O objetivo é detectar se a pessoa inflaciona as respostas positivas para atender às expectativas do pesquisador. $\mathrm{O}$ escore total varia de 33 (baixa desejabilidade social) a 66 (alta desejabilidade social) e apresenta um Coeficiente Alfa de Crombach de 0,76 para a amostra brasileira e de 0,79 para a amostra americana.

\section{Procedimentos}

A pesquisa foi apresentada aos participantes como um estudo sobre resolução de conflitos. Os dados no Brasil foram coletados com amostra e procedimentos semelhantes aos utilizados por Subkoviak et al. (1995) nos Estados Unidos. Para efeito de comparação entre culturas, esses pesquisadores americanos disponibilizaram o seu banco de dados completo ao primeiro autor do presente trabalho. A administração dos instrumentos ocorreu, no caso dos universitários, na própria sala de aula. Quando os estudantes concluíram a participação, receberam um envelope para ser entregue e auto-administrado pelos pais do mesmo sexo. Ou seja, foi solicitado às estudantes mulheres que pedissem a participação de suas mães, enquanto que os estudantes homens foram instados a pedir a participação de seus pais. Nesses envelopes, todas as medidas tinham sido agrupadas e identificadas por números, que relacionavam as amostras de jovens e adultos. Cada envelope continha uma folha de orientação para a auto-administração dos instrumentos pelos pais. Foi solicitado aos estudantes que não discutissem o conteúdo da pesquisa com seus pais antes de todos terem concluído a participação no estudo. A folha de instrução para os pais solicitava também que os envelopes fossem lacrados e retornassem ao pesquisador principal, através do correio ou mesmo do/da filho/a. A presente pesquisa seguiu as orientações éticas estabelecidas pela American Psychological Association 
(APA). Também foi regularmente aprovada pelo comitê de ética da Universidade de Wisconsin-Madison (UWMadison), nos Estados Unidos.

Procedimentos para a Análise dos Dados. Os dados foram analisados pelo primeiro autor, na UW-Madison, utilizando o programa estatístico SPSS para levantar as frequências das variáveis bio-sócio-demográficas, a frequência de respostas ao item do perdão, as médias e os desvios-padrões das subescalas da EFI e da EFI total. Também foram efetuadas análises de confiabilidade interna das escalas EFI e Desejabilidade Social, correlações simples de Pearson entre o item do perdão, os escores da EFI e os escores da desejabilidade social e análises de covariância (ANCOVA), considerando, dentro de cada cultura, o perdão como variável dependente e o sexo, a idade e a religiosidade como variáveis independentes.

\section{Resultados}

As escalas mostraram bons índices de confiabilidade interna, tal como foram reportados na descrição dos instrumentos. Para verificar a validade externa da escala EFI, efetuou-se uma correlação simples de Pearson entre o escore total da EFI e o item do perdão em cada cultura. Os resultados mostraram correlações positivas e significativas para a amostra do Brasil $(n=200, r$ $=0,75, p<0,000)$ e para a amostra dos Estados Unidos $(n=383, r=0,73, p<0,000)$. Correlações simples de Pearson entre o escore total da EFI e o escore da escala de desejabilidade social mostraram não serem significativas na amostra brasileira ou estadunidense. Correlacionando o escore do item do perdão com o escore da escala de desejabilidade social, encontrou-se uma correlação positiva e significativa na amostra brasileira $(n=200, r=0,167, p<0,019)$. Analisando-se esse resultado por categoria de participantes, uma correlação positiva e significativa foi encontrada na amostra de estudantes universitários $(n=100, r=0,247, p<$ $0,014)$ e não na de adultos $(n=100, r=0,128, p<0,204)$, indicando a desejabilidade social nos jovens brasileiros.

Para verificar se o grau de perdão varia em relação à intensidade da mágoa, efetuaram-se correlações simples de Pearson entre o escore total da EFI e o grau de intensidade da mágoa. Foram encontradas correlações negativas e significativas na amostra de brasileiros $(n=200$, $r=-0,243 p<0,001)$ e de estadunidenses $(n=383, r=$ $-0,17, p<0,001)$. Esse resultado indicou que quanto maior o sentimento da mágoa, menor o grau de perdão interpessoal em cada cultura.

No tocante a como o perdão se expressa em graus de afetos, julgamentos e comportamentos em pessoas que reportaram uma grande mágoa ( 45 brasileiros, sendo 23 jovens e 22 adultos e 117 americanos, sendo 57 jovens e 60 adultos), pode-se notar, como mostra a Tabela 2, que em ambas as culturas, a escala de julgamentos da EFI recebeu escore médio maior do que a escala dos afetos e a escala dos comportamentos. A escala dos comportamentos recebeu escore médio intermediário. Para realizar comparações entre as médias das diferentes escalas, por meio de um teste $t$ para amostras relacionadas em cada país, considerou-se que o número de comparações era alto e poderia inflar a possibilidade de resultados significativos que ocorreram ao acaso. Em função disso, dividiu-se o nível esperado de $p \leq 0,05$ pelo número de comparações com teste $t$ (três comparações por grupos de idade) e considerou-se $p \leq 0,017$ como índice aceitável de diferenças significativas. Nos resultados foram observadas diferenças significativas em todas as comparações entre as escalas de afeto e julgamento, indicando que o escore médio da escala de afeto era significativamente inferior ao escore médio da escala de julgamento. Com relação às comparações entre a escala de afeto e a escala de comportamento, verificou-se que ocorreu apenas uma diferença não-significativa referente ao grupo de adultos brasileiros. As demais comparações indicaram que os escores médios da escala do afeto eram inferiores aos da escala do comportamento. Quanto à comparação entre os escores médios das escalas de julgamento e de comportamento, não foram observadas diferenças significativas, com exceção da comparação referente à amostra de adultos brasileiros.

Com relação ao perdão e ao grau de proximidade com quem magôo, os participantes reportaram maior frequência de mágoas entre amigos (Brasil, 32\% e Estados Unidos, 46,3\%). Em seguida, relataram as mágoas na família, ocasionadas por filhos, pais e esposos (Brasil, 25,5\% e Estados Unidos, 22,7\%); as mágoas causadas por parentes, como tios, avós e outros (Brasil, 25,5\% e Estados Unidos, 22,7\%); as mágoas derivadas de estranhos ou outros não-especificados (Brasil, 18\% e Estados Unidos, $8 \%$ ); e, por último, as mágoas entre colegas e chefes de trabalho (Brasil, 3,5\% e Estados Unidos, 9\%). Duas ANOVAs foram realizadas por amostras, considerando o escore do Item do Perdão como variável dependente e os agentes da mágoa (família, parentes, amigos, empregadores e outros) como variáveis independentes. Os resultados com a amostra brasileira mostraram um efeito significativo $\left(F_{4}=3,776\right.$, $p<0,006)$ do agente da mágoa no grau do perdão. O teste LSD de Fischer mostrou que o grau do perdão para mágoas perpetradas por familiares $(n=51, M=3,76$, $D P=1,42)$, parentes $(n=41, M=3,71, D P=1,2) \mathrm{e}$ amigos $(n=64, M=3,81, D P=1,15)$ era maior do que o grau do perdão para mágoas ocasionadas por empregadores ou colegas de trabalho $(n=7, M=2,0, D P=$ $1,3)$. Além disso, o grau do perdão para mágoas engendradas por empregadores era significativamente menor do que o perdão de mágoas causadas por outros sem especificação $(n=36, M=3,39, D P=1,25)$.

Os resultados na amostra americana também mostraram um efeito significativo $\left(F_{4}=15,067, p<0,000\right)$ do agente da mágoa, no grau do perdão. O teste LSD de 
Tabela 2

Testes t para Subescalas da EFI - Amostras Relacionadas por uma Grande Intensidade de Mágoa e Idade

\begin{tabular}{|c|c|c|c|c|}
\hline Pais/Subescala & $M(D P)$ & $t$ & $\mathrm{gl}$ & Sig. \\
\hline \multicolumn{5}{|l|}{ BRASIL $(n=45)$} \\
\hline \multicolumn{5}{|l|}{ Jovens $(n=23)$} \\
\hline Afeto x Julgamento & $74,0(31,0) \times 85,0(29,0)$ & $-4,439$ & 22 & 0,000 \\
\hline Afeto x Comportamento & $74,0(31,0) \times 81,0(29,0)$ & $-2,593$ & 22 & 0,017 \\
\hline $\begin{array}{l}\text { Comportamento x Julgamento } \\
\text { Adultos }(n=22)\end{array}$ & $81,0(29,0) \times 85,0(29,0)$ & $-1,752$ & 22 & 0,094 \\
\hline Afeto x Julgamento & $67,0(27,0) \times 80,0(27,0)$ & $-4,518$ & 21 & 0,000 \\
\hline Afeto x Comportamento & $67,0(27,0) \times 73,0(25,0)$ & $-1,841$ & 21 & 0,080 \\
\hline Comportamento x Julgamento & $73,0(25,0) \times 80,0(27,0)$ & $-2,598$ & 21 & 0,017 \\
\hline \multicolumn{5}{|l|}{ ESTADOS UNIDOS $(n=117)$} \\
\hline \multicolumn{5}{|l|}{ Jovens $(n=57)$} \\
\hline Afeto x Julgamento & $72,0(31,0) \times 87,0(25,0)$ & $-6,444$ & 56 & 0,000 \\
\hline Afeto x Comportamento & $72,0(31,0) \times 85,0(28,0)$ & $-6,865$ & 56 & 0,000 \\
\hline $\begin{array}{l}\text { Comportamento x Julgamento } \\
\text { Adultos }(n=60)\end{array}$ & $85,0(28,0) \times 87,0(25,0)$ & $-1,152$ & 56 & 0,254 \\
\hline Afeto x Julgamento & $69,0(30,0) \times 82,0(26,0)$ & $-5,213$ & 59 & 0,000 \\
\hline Afeto x Comportamento & $69,0(30,0) \times 80,0(27,0)$ & $-5,905$ & 59 & 0,000 \\
\hline Comportamento x Julgamento & $80,0(27,0) \times 82,0(26,0)$ & $-1,095$ & 59 & 0,278 \\
\hline
\end{tabular}

Fischer mostrou que o grau do perdão para mágoas perpetradas por familiares mais próximos $(n=86, M=4,00$, $D P=1,15)$ era significativamente maior do que o grau do perdão para mágoas geradas por parentes $(n=54, M$ $=3,31, D P=1,15)$, amigos $(n=179, M=3,59, D P=$ $1,15)$, empregadores $(n=35, M=2,77, D P=1,24) \mathrm{e}$ outros sem especificação $(n=31, M=2,35, D P=1,35)$. O grau do perdão para mágoas perpetradas por parentes era significativamente menor do que o grau do perdão para os familiares mais próximos. Porém, era significativamente maior do que o grau do perdão para empregadores e outros, além de não se diferenciar do grau do perdão oferecido aos amigos. Não existiram diferenças significativas no grau do perdão para mágoas perpetradas por empregadores e outros.

Para verificar o efeito do sexo, idade e religiosidade no escore total da EFI, foi efetuada, para cada país em separado, uma ANCOVA considerando o perdão como variável dependente e as demais variáveis como covariantes. Os resultados na amostra brasileira não mostraram efeito significativo do sexo, idade ou religiosidade no perdão. $\mathrm{Na}$ amostra estadunidense, o resultado foi significativo $\left(F_{4}=2,982, p \leq 0,019\right)$ indicando um efeito da religiosidade $\left(F_{1}=5,323, p \leq 0,022\right)$ no perdão, que explicou $0,03 \%$ da variância total.

\section{Discussão}

As escalas utilizadas mostraram-se válidas e confiáveis para estudar o perdão interpessoal. No tocante ao perdão ser oferecido de forma espontânea e completa, confir- mam-se as expectativas teóricas de Enright (Enright \& the Human Development Study Group, 1991), no sentido de que para perdoar é preciso reconhecer que existiu uma mágoa e é a percepção dessa mágoa que vai indicar a intensidade da indignação. Consequentemente, espera-se, dentro dos padrões normais do desenvolvimento humano, que após uma grande mágoa seja mais difícil o perdão ser oferecido espontânea e completamente. Nesse sentido, os resultados da literatura empírica com amostras americanas (Subkoviak et al., 1995) mostraram que o perdão interpessoal é oferecido como uma função da percepção da mágoa. Os resultados encontrados com as amostras brasileiras apóiam uma maior generalização dessa expectativa, de que pessoas que foram magoadas fortemente chegam a considerar o perdão, mas não perdoam completamente.

Em relação à proximidade no relacionamento com o ofensor, perguntou-se se as pessoas perdoam mais os familiares e os amigos do que os estranhos e os colegas de trabalho. Rique et al. (2007) já informaram que o perdão varia em relação à percepção da mágoa sofrida e em relação à proximidade no relacionamento com o ofensor. Ou seja, considerando que diante de mágoas fortes o grau de perdão concedido torna-se menor, ainda assim as pessoas perdoam os membros da família e os amigos em maior grau do que perdoam os estranhos ou os colegas de trabalho.

Considerando-se apenas às mágoas no contexto da família, na amostra americana verificou-se que os familiares mais próximos (pais, filhos e esposos) receberam maior consideração pelo perdão do que os parentes (tios, 
avós e outros), que ficaram com média semelhante à dos amigos. No Brasil, não ocorreu essa distinção dentro da família. Comumente, é dito que a família brasileira promove maior aproximação e maiores vínculos entre avós, tios e primos e os resultados confirmam essa expectativa.

No contexto das amizades, os resultados indicam que os amigos começam a fazer parte dessa rede de vínculos afetivos, mesmo sem a consanguinidade. Tanto no Brasil quanto nos Estados Unidos, os amigos recebem um grau de perdão tão elevado quanto os familiares.

$\mathrm{Na}$ amostra brasileira, chama a atenção o fato de que as mágoas perpetradas por estranhos ou outros receberam um grau de perdão maior do que o perdão concedido a empregadores ou colegas de trabalho. Na amostra americana, não ocorreram distinções entre colegas de trabalho e outros. Se, por um lado, não é difícil compreender que o perdão seja normativo nas relações familiares e de amizades, dependendo do grau da mágoa, por outro lado, não é fácil perceber o que ocorre com relação aos colegas de trabalho e aos empregadores. A questão fica para futuros esclarecimentos. Mais do que afirmar que as mágoas interpessoais são intransponíveis no contexto de trabalho, é preciso entender primeiro a natureza dessas mágoas e a qualidade do vínculo que se estabelece entre colegas de trabalho, para então se ousar interpretar o perdão.

Com relação aos afetos, julgamentos e comportamentos para com a pessoa que ofendeu, observaram-se diferenças significativas entre afeto e julgamento e entre afeto e comportamento, que foram semelhantes para os brasileiros e para os estadunidenses. Em média, o grau de perdão pelo afeto permaneceu significativamente inferior ao grau do perdão pelos julgamentos. Reforçou-se aqui a idéia de que se torna mais difícil para as pessoas perdoar afetivamente (ex., voltar a amar o ofensor) do que cognitivamente (ex., elaborar julgamentos positivos sobre o ofensor). Este resultado remete ao modelo anteriormente verificado por Rique et al. (in press), onde se verificou que o perdão se expressa através da presença de elementos positivos nos afetos, julgamentos e comportamentos e da ausência dos elementos negativos nesses mesmos domínios das atitudes. O que se supõe, agora, é que as modificações no aumento dos aspectos positivos não ocorrem com a mesma força que a redução dos aspectos negativos, principalmente no tocante aos afetos. Trata-se de saber se, quando uma pessoa perdoa completamente, os escores dos afetos positivos pelo ofensor permanecem inferiores aos escores do julgamento, ou se isto ocorreu porque as pessoas não reportaram um perdão completo. Essa é uma questão que por certo impulsionará a realização de novos estudos.

Por último, no que tange à influência da cultura no perdão, os resultados mostraram que não ocorreram diferenças significativas no grau de perdão entre as amostras do Brasil e dos Estados Unidos. Adianta-se aqui que os estudos realizados em três outros países, Áustria,
Israel e Taiwan, nos quais a EFI foi traduzida e administrada em amostras semelhantes às utilizadas neste estudo, também indicam que o perdão não se diferencia significativamente entre culturas. Espera-se que os resultados de análises que estão comparando o modelo do perdão em seis culturas, possibilitem entender e discutir melhor o perdão a nível transcultural.

Quanto à influencia do sexo, idade e afiliação religiosa no perdão, os resultados não mostraram diferenças significativas. Como informado na revisão da literatura, pesquisadores (Park, 1998; Subkoviak et al., 1995) que utilizaram instrumentos e metodologia semelhantes aos deste estudo verificaram que, em alguns contextos, ocorrem diferenças de sexo no perdão. Também se esperava que, com o amadurecimento afetivo no desenvolvimento, aqui representado pela idade, o perdão fosse oferecido em maior grau. Os resultados não confirmaram essa expectativa. A questão que fica é se o perdão depende de modificações nos valores ou na visão de mundo (ideologias) ao longo da vida, ou seja, não é simplesmente amadurecer, mas mudar os valores ao longo do amadurecimento.

Em conclusão, o presente estudo replica resultados reportados na literatura, porém os resultados são limitados a amostras colhidas no âmbito da educação universitária e com adultos que são pais dos participantes universitários. Embora o delineamento do presente trabalho ajude a obter dados de duas gerações, os valores serão sempre semelhantes, pois os dados são coletados dentro da mesma família ou grupo. Portanto, recomenda-se que futuros estudos investiguem o perdão em amostras de outros países, além do Brasil e dos Estados Unidos e em outras regiões de cada país, pois é relevante se obter uma normatização do grau de perdão dentro de uma cultura. Deve-se também coletar dados em outros grupos dentro de uma mesma região, como, por exemplo, verificar o perdão em adolescentes infratores em centros de ressocialização na região nordeste, o perdão em idosos institucionalizados, entre outros. Obter a normatização do grau do perdão em uma cultura e os graus de perdão em grupos específicos, facilitará a realização de trabalhos de avaliação psicológica e de intervenções, no sentido de favorecer um melhor equilíbrio psicológico nas pessoas e uma maior harmonia das relações sociais.

\section{Referências}

Arendt, H. (1998). The human condition. Chicago, IL: The University of Chicago. (Original work published 1958).

Butler, J. (2002). Sermon VIII: Upon resentment and forgiveness of injuries (L. R. Dagg, Trans.). In J. Butler, Fifteen Sermons: Preached at the rolls chapel. (Original work published 1827). Retrieved July 18, 2009, from http:// anglicanhistory.org/butler/rolls/index.html

Crowne, D. P., \& Marlowe, D. (1960). A new scale of social desirability independent of psychopathology. Journal of Consulting Psychology, 24, 349-354. 
Digeser, P. E. (2001). Political forgiveness. Ithaca, NY: Cornell University Press.

Enright, R. D., \& Fitzgibbons, R. P. (2001). Helping clients forgive: An empirical guide for resolving anger and restoring hope. Washington, DC: American Psychological Association.

Enright, R. D., Freedman, S., \& Rique, J. (1998). The psychology of interpersonal forgiveness. In R. D. Enright \& J. North (Eds.), Exploring forgiveness (pp. 46-62). Madison, WI: University of Wisconsin Press.

Enright, R. D., \& Human Development Study Group (1991). The moral development of forgiveness. In W. Kurtiness \& J. Gerwitz (Eds.), Handbook of moral behavior and development (Vol. 1, pp. 123-152). Hillsdale, NJ: Erlbaum.

Enright, R. D., \& Human Development Study Group (1994). Piaget on the moral development of forgiveness: Reciprocity or identity? Human Development, 37, 63-80.

Enright, R. D., \& Rique, J. (2001). The Enright Forgiveness Inventory: User's manual. Menlo Park, CA: Mind Garden Press.

Holter, A. C., Martin, J., \& Enright, R. D. (2003). Restoring justice through forgiveness. The case of children in Northern Ireland. In D. Sullivan \& L. Tifft (Eds.), Handbook of restorative justice (pp. 311-320). New York: Routledge.

McKnight, A. N. (2004). Historical trauma, the persistence of memory and the pedagogical problems of forgiveness, justice and peace. Educational Studies, 36(2), 140-158.

Murphy, J. G., \& Hampton, J. (1998). Forgiveness and Mercy. New York: Cambridge University. (Original work published 1988)

Nussbaum, M. (1998). Sex and social justice. New York: Oxford University.

Park, S. (1998). Measuring forgiveness across three cultures. Unpublished doctoral dissertation, University of WisconsinMadison, WI.

Reed, G. L., \& Enright, R. D. (2006). The effects of forgiveness therapy on depression, anxiety and posttraumatic stress for women after spousal emotional abuse. Journal of Consulting and Clinical Psychology, 74(5), 920-929.

Rique, J. (1999). A cross-cultural study on the Enright Forgiveness Inventory: A measure for interpersonal forgiveness. Samples from Brazil and the United States. Unpublished doctoral dissertation, University of WisconsinMadison, WI.

Rique, J., Camino, C., Enright, R. D., \& Queiroz, P. (2007). Perdão interpessoal em contextos de injustiças. Psico, 38(2), 185-192.

Rique, J., Camino, C., Santos, W. S., \& Gouveia, V. V. (2009). Análises confirmatórias da Escala de Atitudes para o Perdão - EFI. Revista Avaliação Psicológica, 8(2), 169-178.

Seligman, M. E. P., \& Csikszentmihalyi, M. (2000). On happiness, excellence, and optimal human functioning [Special issue]. American Psychologist, 55(1).

Subkoviak, M. J., Enright, R. D., Wu, C., Gassin, E., Freedman, S., Olson, L., et al. (1995). Measuring interpersonal forgiveness in late adolescence and middle adulthood. Journal of Adolescence, 18, 641-655.

Worthington, E. L., Jr. (1998). Dimensions of forgiveness: Psychological research \& theological perspectives. Radnor, PA: Templeton Foundation.

Worthington, E. L., Jr. (2005). Handbook of forgiveness. New York: Routledge. 